\title{
What Makes Babies Musical? Conceptions of Musicality in Infants and Toddlers
}

\author{
Verena Buren ${ }^{1 *}$, Daniel Müllensiefen ${ }^{2}$, Tina C. Roeske ${ }^{1}$ and Franziska Degé1 \\ ${ }^{1}$ Department of Music, Max Planck Institute for Empirical Aesthetics, Frankfurt/M, Germany, ${ }^{2}$ Department of Psychology, \\ Goldsmiths, University of London, London, United Kingdom
}

Despite major advances in research on musical ability in infants, relatively little attention has been paid to individual differences in general musicality in infants. A fundamental problem has been the lack of a clear definition of what constitutes "general musicality" or "musical ability" in infants and toddlers, resulting in a wide range of test procedures that rely on different models of musicality. However, musicality can be seen as a social construct that can take on different meanings across cultures, sub-groups, and individuals, and may be subject to change over time. Therefore, one way to get a

OPEN ACCESS

Edited by:

Eckart Altenmüller,

Hanover University of Music Drama and Media, Germany

Reviewed by:

Miriam Lense,

Vanderbilt University, United States

McNeel Gordon Jantzen,

Western Washington University,

United States

*Correspondence: Verena Buren

verena.buren@ae.mpg.de;

verena.buren@gmx.de

Specialty section:

This article was submitted to Auditory Cognitive Neuroscience,

a section of the journal

Frontiers in Psychology

Received: 05 July 2021

Accepted: 19 November 2021

Published: 15 December 2021

Citation:

Buren V, Müllensiefen $D$ Roeske TC and Degé $F$ (2021) What Makes Babies Musical? Conceptions of Musicality in Infants and Toddlers.

Front. Psychol. 12:736833.

doi: 10.3389/fpsyg.2021.736833 clearer picture of infant musicality is to assess conceptions of musicality in the general population. Using this approach, we surveyed 174 German adults, asking about their view and conceptions regarding behaviors that characterize a musical child under 3 years. Based on previous studies on adult and child musicality, we designed a survey containing 41 statements describing musical behaviors in children. Participants were asked to rate how indicative these behaviors were of musicality in infants and toddlers. PCA analysis revealed 4 components of musical abilities and behaviors in under-3-yearolds: Musical Communication, Enthusiasm and Motivation, Adaptive Expressiveness, and Musical Abilities as traditionally defined. Professional background and musical expertise of the respondents did not significantly influence participants' conceptions. Our results suggest that, in order to capture musicality in young children, a wider range of skills and observable behaviors should be taken into account than those assessed by traditional musical ability tests for young children.

Keywords: musicality, musical ability, conceptions of musical ability, development of musical ability, survey, infants, toddlers, musical development

\section{INTRODUCTION}

Across the last century, a wide range of tests have been developed to measure musical ability in childhood (e.g., Gordon, 1965, 1979, 1982, 1989a,b; Bentley, 1966; Seashore, 1967; Wing, 1981). Strikingly, these instruments are based on a very diverse range of different theoretical models and conceptions of musical ability (Shuter-Dyson, 1999). This diversity is due to the fact that musicality is not a natural category, but a social construct that can take on different meanings in different cultures, subgroups, and even individuals (Blacking, 1971; Hallam and Prince, 2003). We here use the term musicality to describe a broader understanding of musical ability that includes not only musical perception and production, but also emotional responses to music, emotional 
expressiveness through music, and interest and motivation in musical activities. In contrast to the term musicality, the term musical ability is often associated with musical perception or production skills assessed by traditional behavioral tests. The social construct of musicality cannot be assessed directly because it can manifest itself through a wide range of observable behaviors with relation to music. Therefore, it is necessary to identify indicators that reliably reflect the construct. The identification of behavioral indicators of musicality can lead to a broader understanding of how (conceptions of) musicality change(s) across different target ages and serve as a starting point for the development of age-appropriate test procedures, which can then be used to determine early musical skills and to map individual developmental trajectories.

In order to investigate musical development from the very beginning, it is essential to capture the first signs of musicality. Early observations by parents and educators can provide important information about which behaviors are perceived as the first indicators of musicality. Therefore, the objective of the present study was to investigate conceptions of infant/toddler musicality as perceived by adults in Germany with different professional backgrounds and musical expertise. The aim was to determine which abilities and behaviors in infants and toddlers are interpreted as indicators of musicality. This knowledge will enable us to deepen our understanding of what constitutes musicality in the first years and to develop a clearer picture of early musicality and its facets. The results of this study may help to explain how musicality can be described in infants and toddlers and thus provide a basis for developing appropriate measurement procedures in the future.

\section{The Development of Musical Abilities}

Music is a cultural universal (Merriam, 1964; Blacking, 1995) and all humans are assumed to possess a potential for musical competence (Trehub et al., 2015a). Current research suggests that the development of musicality begins in the womb and continues into adulthood (Gooding and Standley, 2011) through processes of enculturation and musical training (Hannon and Trainor, 2007).

\section{Musical Development in the First Years of Life}

Already in the first months of life, children can develop amazing perceptual abilities (Trehub, 2003). For example, sensitivity to melodic contours and relative pitches are developed in early infancy (Trehub, 2015). Moreover, certain perceptual abilities that are central to music cognition are initially universal, i.e., infants have the perceptual prerequisites to recognize the musical subtleties of any musical culture (Trehub, 2015). However, over time, culture-specific attunement takes place, and children develop more and more culture-specific skills (Trehub, 2015). An example is rhythm perception, where 6month-old infants show a culture-general pattern of responding to musical rhythms, whereas 12-month-old infants already show an adult-like, culture-specific pattern of responding (Hannon and Trehub, 2005).

Rhythm production skills also develop rapidly in early childhood. Although two-and-a-half-year-olds can already adapt the tempo of their drumming to that of a drumming partner (Kirschner and Tomasello, 2009), synchronous drumming with a rhythmic pattern is not achieved until around the age of 4 (Provasi and Bobin-Bègue, 2003). Other skills (such as sensitivity to harmony) do not develop until later childhood (e.g., Schellenberg et al., 2005).

Infants are also generally attracted to music and musiclike stimuli (like infant-directed speech) from a very early age and give carefully matched expressive responses. By doing this, they create co-operative patterns of communication that can be interpreted as a form of communicative musicality (Malloch, 2000; Flohr and Trevarthen, 2008), in which early singinglike and speaking-like vocalizations are still indistinguishable (Stadler Elmer, 2012). After their first birthday, children are increasingly able to express themselves musically: they dance to music in a rudimentary way (Trehub, 2015), and a few months later, their early vocalizations differentiate into speaking and singing, with early singing showing characteristic features such as glissandi, unstable pitches, singing with indefinable sounds, neologisms, short phrases in a narrow vocal range, and small and imprecisely tuned intervals (Stadler Elmer, 2012). Thus, while rudimentary singing is already present in under 3-year-olds, children become more sophisticated between the ages of 3 and 4 and begin to combine songs or song fragments. They hereby increasingly succeed in approximating the phrase contours of a song (Gembris, 2017), until the development of singing ability is largely complete in 8-year-olds (Davidson, 1994).

\section{The Musical Environment of Babies}

Especially in early childhood, parents and their musical practices in the home environment have a major influence on children's musical lives (Ilari, 2018). One of the first and most common musical interactions with babies is singing (Ilari, 2005; Young, 2008). When singing to their infants, parents sing in a distinctive manner [e.g., by using high pitch, slow tempo, and more expressive rendering of lyrics (Trehub et al., 1997a,b)]. This socalled infant-directed singing is often used to modulate infant arousal (Shenfield et al., 2003; Trehub et al., 2015b) and it also serves social bonding functions (Trehub, 2015). Especially in musical interactions with babies, singing is an important way of musical interaction with the caregiver (Ilari, 2005; CostaGiomi and Ilari, 2014). Additionally, the special importance of the voice has also been demonstrated in a series of studies which have shown that sung melodies are better remembered than instrumental melodies (Weiss et al., 2012, 2015, 2021). Besides singing, many parents rely on recorded music or modern toys with musical features to create a musical environment for their children (de Vries, 2009; Mehr, 2014). Although the use of recorded music offers many advantages (e.g., the ability to engage with music without a parent, the ability to offer a greater variability of styles), recorded music lacks the multimodality of live interaction with a caregiver. In addition, there seems to be a widespread belief that educational settings provide children with a more comprehensive musical experience (de Vries, 2009) which may lead parents to think that their own musical interactions are of lesser importance. This suggests that parents' goals and beliefs about their children's musical development are very influential, 
as they affect the styles and practices they use with their children (McPherson, 2009). Therefore, it is important to define musical development not only from current theories of human development, but also to consider musicality as a social construct that is influenced by contemporary discourses in music education research, by different approaches in educational practice, and by common believes about child musicality as held by parents, carers, and educators.

\section{Assessing Musicality in Childhood}

There are already many findings on "age-related stages of mastery of basic elements of the Western-European music system" (Stadler Elmer, 2011, p. 13). Thus, many studies examine skills that are present on average at a given age in order to draw conclusions about common developmental trajectories (Stadler Elmer, 2011). However, this can lead to glossing over or omitting important dimensions/indices of musical development (Forrester and Borthwick-Hunter, 2015). Although this approach may show that a skill or competence has emerged, it can easily fail to capture the causes, context and important associations of its emergence (Forrester and Borthwick-Hunter, 2015). A more comprehensive approach requires the consideration of more complex behaviors in culturally embedded settings (as has been shown for early singing development; see Stadler Elmer, 2011, 2012). The extension and integration of the existing findings and the implementation of a test battery based on these findings to monitor musical developmental processes could be a long-term goal of research on musical development.

While developmental science has made great progress in assessing how and when different basic musical abilities develop (see Trehub and Hannon, 2006; Trainor and Unrau, 2011; Trehub, 2015, for comprehensive reviews on this topic), relatively little progress has been made toward understanding how these abilities relate to each other and to the conception of general musicality. To study the development of general musicality adequately, we need scientific measurement tools that have a solid basis in developmental theory as well as being compatible with the conceptions of musicality of important stakeholders (i.e., general and music educators, carers, parents).

One approach to the study of musical ability is the use of standardized tests. However, these cannot be used with children under 3 years of age. Additionally, the traditional tests used in later childhood [such as the Seashore Measures of Musical Talent (Seashore, 1919), the Measures of Musical Abilities developed by Bentley (1966), or the "Gordon tests" (Gordon, 1965, 1979, 1982, 1989a,b)] place an emphasis on auditory and receptive skills and largely neglect music production behavior (composing, improvising, playing an instrument, or singing), motivation, and emotional and communicative elements (such as aesthetic responses, musical communication, and expressiveness). Thus, these measures are unlikely to be sufficient to provide a comprehensive picture of musicality and its development (Murphy, 1999). In addition, before assessing a construct, it is useful to clearly define which components are to be assessed in the first place (which requires a clear definition of musicality and its different facets). Some researchers have even questioned whether a single test of musical ability can capture the manifold manifestations of musicality at all (Murphy, 1999), which is especially true for infants and young children. Therefore, measurement instruments need to be highly adapted or supplemented with age-appropriate observational instruments based on a clear definition of musicality.

Parent reports can therefore be a valuable source for the study of musical behaviors in young children. An example is the Children's Musical Behavior Questionnaire (Valerio et al., 2012), which surveys music-related behaviors (as documented by parents about children and themselves). It includes 97 items aimed at finding out how often children exhibit a range of musical behaviors (e.g., singing, dancing, or listening to music). Seven factors relate to child-initiated musical behaviors (Attention and Emotion, Vocalizations, Moving, Daily Routines, Requests, Taking Turns, Creativity) and one relates to parentinitiated activities.

An even more comprehensive survey is the Music@home questionnaire (Politimou et al., 2018) which was designed to systematically map musical engagement in the home environment of young children. The infant version (323 months) of the Music@Home scale examines four factors: Parental beliefs (i.e., what parents think about music and development), Child engagement with music, Parent initiation of singing, and Parent initiation of music-making. In the Preschool version (2-5.5 years) the four factors were Parental beliefs, Child engagement with music, Parent initiation of singing, and Breadth of musical exposure.

Questionnaires such as the Children's Musical Behavior Questionnaire and the Music@home questionnaire are valuable for identifying musical opportunities and behaviors in infants and young children. They enable the empirical documentation of musical behaviors that children exhibit in their home environment and how parents use their musical nurturing opportunities. In addition, they take advantage of primary caregivers' rich knowledge about their children's development, which cannot always be adequately accounted for in laboratory studies. However, they do not extensively elucidate what conceptions of musicality underlie the behaviors exhibited. Moreover, interindividual differences and comparisons across different trajectories of musical development are much less a focus of these questionnaire and survey instruments.

\section{Conceptions of Musicality}

Approaching musicality as a social construct, Hallam and colleagues examined how people with different musical experiences conceptualize musicality (Hallam and Shaw, 2002; Hallam and Prince, 2003; Hallam, 2010; Hallam and Papageorgi, 2016). In a first qualitative study, Hallam and Prince (2003) asked 415 participants (adults and adolescents) from the United Kingdom to complete in writing the statement "musical ability is...". In an iterative process of categorization, the collected statements were analyzed and six overarching themes were identified (aural abilities, receptive responses, generative activities, integration of a range of abilities, personal characteristics, whether musical abilities are innate or learned). 
Overall, the statements contained more references to active music making than to receptive skills and became more complex as respondents' musical experience increased.

The 77 statements derived from the qualitative study then served as a starting point for the quantitative study of musical conceptions (Hallam, 2010). Six hundred and sixty participants (musicians, educators, amateur musicians, children with and without musical engagement) rated these statements according to their level of agreement. Through principal component analysis, the authors identified six components of musicality: (1) playing an instrument or singing, (2) musical communication (communicating emotions through music), (3) valuing, appreciating, and responding to music, (4) composition, improvisation, and related skills, (5) commitment, motivation, personal discipline and organization, (6) rhythmic ability, pitch, and understanding. The conceptions differed between participants depending on their professional and musical experiences: Musical communication was perceived as the most important ability by musicians, whereas educators placed more emphasis on creativity. In contrast, amateur musicians and nonmusicians held the belief that musicality is constituted by high aural skills and motivation.

In order to investigate whether these conceptions of musicality in general also apply to conceptions of musicality in childhood, the statements of the original study by Hallam and Prince (2003) were adapted so that they corresponded in principle to the abilities of the relevant age group (in this case, 3- to 6year-olds; Buren et al., 2021). Nine hundred and twenty-two adults in Germany (music educators and musicians, educators but without specialization in music, parents and carers) rated the 49 resulting statements according to how frequently they thought a musical child between the ages of 3 and 6 exhibited these behaviors. Similar to Hallam's (2010) results, a differentiated and multifaceted picture of musicality emerged. Through PCA analysis, four components of children's musical abilities and behaviors were identified: (1) Musical Communication, (2) Enthusiasm and Motivation (the affinity and the enjoyment of music and the motivation to make music), (3) Analytical Understanding (the awareness of different aspects of music and the use of this knowledge to evaluate music), and (4) Musical Abilities (audiation abilities and the integration of these abilities for making music). On average, participants rated enthusiasm and motivation as the most important indicators of musicality in childhood, followed by musical abilities like audiation skills. Musical and educational training of the survey participants only affected the ratings on the component Analytical Understanding. The analytical understanding of 3- to 6-year-olds was considered more important by parents and carers than by educators, while music educators and musicians attributed the least importance to this component.

Since children under the age of 3 years have specific cognitive, motor, and emotional prerequisites (Bukatko and Daehler, 2004; Siegler et al., 2014; Berk, 2018), it remains unclear whether the conceptions of musicality in childhood (between 3 and 6 years) extend to children under the age of 3 years. Hence, it seems highly likely that adults would assess musicality in children under 3 years of age according to differing criteria.
For example, in terms of the component Musical Communication, it is unclear whether it is seen as equally important in children under 3 years of age, because musical vocalizations before 2 years of age are hard to distinguish from preverbal vocalizations (Stadler Elmer, 2012). Since the components Analytical Understanding and Musical Abilities are also heavily influenced by basic cognitive and linguistic skills, it remains to be seen whether they are also considered important for conceptions of musicality in children under 3 years. Furthermore, the component of musical abilities may also play a different role in younger children, since musical production skills in particular (e.g., tapping, drumming) are strongly dependent on age-related motor development processes.

The aim of this study was to draw a more comprehensive picture of infant musicality which will allow us to develop meaningful, reliable, and objective test procedures for musicality that build on a clear operational definition of the construct being measured. In addition to approaching musicality from a theoretical perspective, the empirical approach pursued in this study can confirm and complement assumptions derived from theories on musical development (e.g., Swanwick and Tillman, 1986; Serafine, 1988; Hargreaves and Galton, 1992; Gordon, 2007; Ockelford, 2013). The everyday experience of parents and educators can provide added value that can serve to refine theories. Theoretical approaches, such as general factor or multifactorial approaches, serve to systematize in a meaningful way, but should also stand up to empirical scrutiny.

In the present study, we investigated how German adults from different professional backgrounds and with a range of musical expertise perceive the construct musicality in early childhood. More specifically, we were interested in their assessment of behavioral indicators and personal factors that might be considered expressions of musicality. To this end, we created a questionnaire that contained statements about musical abilities and behaviors and asked participants with varying professional and musical backgrounds to rate these statements, according to how well they thought the statement described musicality in under-3-year-olds. The results of this study can serve as a basis for the development of a musicality test that does not reduce musicality to individual aspects, but reflects a more comprehensive understanding of musical behavior.

\section{MATERIALS AND METHODS}

\section{Participants}

The sample comprised 221 participants between 20 and 69 years $(M=38.97, S D=8.89)$ who indicated that they regularly spend time with children under the age of 3 years. Participants were excluded if they had filled in less than $50 \%$ of the questionnaire items $(n=29)$, if they had given constant ratings to all items $(n=9)$, or if they took less than 4 minutes to complete the entire survey $(n=9)$, resulting in a sample of 174 valid cases. Out of those, 146 were female and 66 had a university degree (of which $n=21$ had a degree in music), 50 had the equivalent of A-levels, 50 had the equivalent of a general secondary school leaving certificate and 8 had a lower secondary school leaving 
certificate or no certificate at all. The final sample included 76 early childhood educators and 96 parents and carers without professional training in pedagogy or music education. Two participants did not indicate their profession.

\section{Materials \\ The Musical Child Questionnaire for Infants and Toddlers (MCQ_U3)}

The Musical Child Questionnaire for children under 3 years (MCQ_U3) is an adapted version of the Musical Child Questionnaire (MCQ), which was developed to assess conceptions of musicality in children between 3 and 6 years of age (Buren et al., 2021). The basis for the development of both questionnaires was a survey by Hallam and Prince (2003) where participants had to complete the sentence: "musical ability is..." Statements were translated into German and adapted for the use with children from 0 to 3 years by adjusting to the ability spectrum of this age group. For example, the phrase "being able to move in time with a rhythm" (Hallam and Prince, 2003) was transformed to "A musical child under 3 years is able to move according to the music." All decisions regarding the reformulation or elimination of items were made by consensus of all authors. The final version of the Musical Infant/Toddler Questionnaire consisted of 41 statements describing musical abilities and behaviors in infants and toddlers. Participants rated on a 5-point-Likert-scale (ranging from $1=$ rarely/never to 5 = always) how often a child under the age of 3 years that they considered as "musical" would typically show these behaviors.

\section{The Goldsmiths Musical Sophistication Index}

To assess the musical background of all survey participants, they were asked to complete the seven-item musical training subscale of the Goldsmiths Musical Sophistication Index (Gold-MSI; Müllensiefen et al., 2014) in its German version (Schaal et al., 2014).

\section{Demographic Information}

All participants provided information about their age, gender, educational background, and profession.

\section{Procedure}

Data were collected between April 2019 and June 2020 in Germany. Most of the respondents were recruited online using e-mail distribution lists from music schools, day care centers and the panel of a market research agency. The study was approved by the Ethics Council of the Max Planck Society, and informed consent was obtained from all participants.

\section{Analytic Strategy}

Data analyses were performed using the R software environment (R Core Team, 2019), including the $\mathrm{R}$ packages psych (Revelle, 2020) and lavaan (Rosseel, 2012).

The main goal of the data analyses was to identify distinguishable facets of musical abilities and behaviors in infants/toddlers, and to compare these to the facets of musical behaviors previously identified in children between 3 and 6 years (Buren et al., 2021). As a first step, the grouping structure of the data was explored by computing a series of principal component analyses (PCA) and comparing solutions to the model previously established for children from 3 to 6 years by confirmatory factor analysis.

In a second step, a shorter scale of the MCQ_U3 with better psychometric properties was constructed using PCA. Differences in scale scores were then investigated with respect to participants' levels of musical or educational training through correlations and multivariate analysis of variance.

\section{RESULTS}

\section{Descriptive Analysis}

Table 1 lists the top 10 items that were rated by the participants as the most frequent indicators of musicality in children under 3 years of age. Interestingly, the seven items with the highest mean values are similar in content. These statements describe a high interest in music, enjoyment of music and the motivation to become musically active. The three remaining items in the top 10 list describe that a musical child has individual preferences in music taste and a "musical ear."

\section{Grouping Items of the MCQ_U3}

We computed three PCA models to group the items of the MCQ_U3 into different components. The first grouping was computed following Hallam's (2010) analysis strategy, retaining all PCA components with Eigenvalues above 2 and applying varimax rotation. The resulting model comprised 2 components and explained $44 \%$ of the variance (see Table 2 for the root mean square of the residuals). As an alternative, parallel analysis (Horn, 1965) was used to determine the number of PCA components which generated a 2-component solution to which oblimin rotation was applied. The resulting model also explained $44 \%$ of variance. Last, we specified a 4-component model with oblimin rotation to investigate similarities to the model previously established for children from 3-6 years (Buren et al., 2021). This solution explained 52\% of the item variance.

All three models were compared using confirmatory factor analysis along with the previously established model for 3-6year-olds (Buren et al., 2021). Fit indices are shown in Table 2.

Both 2-component-solutions were highly similar in content as well as in their fit indices. The 4-component PCA model proved to have the best model-data-fit, according to the Bayesian information criterion (BIC). Because both the relative (TLI, CFI) and absolute fit indices (RMSEA, SRMR) for this model were also in an acceptable to good range, we decided to select the 4-component-model for content interpretation (see Table 3 for items and their loadings).

The first component included items that reflected types of musical communication (e.g., "A child under 3 years who is musically skilled can communicate with others through music by producing musical sounds, listening, improvising, dancing, and understanding music"), or musical understanding (e.g., "A child under 3 years who is musically skilled shows that he/she can capture patterns when dancing or 
TABLE 1 | Highest rated items of the Musical Infant/Toddler Questionnaire (MCQ_U3).

\begin{tabular}{|c|c|c|c|}
\hline Item no. & A child under 3 years who is musically skilled... & $M$ & $S D$ \\
\hline 43_U3 & ... enjoys the occupation with music. & 4.20 & 0.78 \\
\hline 38_U3 & $\ldots$ is interested in music. & 4.16 & 0.85 \\
\hline 41_U3 & ... has great enthusiasm for music. & 4.09 & 0.81 \\
\hline 14_U3 & ... enjoys music in his/her life, either by making music or by listening to it. & 4.05 & 0.87 \\
\hline 45_U3 & ... has a great affinity for music. & 3.94 & 0.84 \\
\hline 15_U3 & ... enjoys music and appreciates sounds. & 3.88 & 0.89 \\
\hline 29_U3 & $\begin{array}{l}\text {... has the desire to make music together with others so that they become a group-either by singing along or moving } \\
\text { to the music of others. }\end{array}$ & 3.76 & 0.99 \\
\hline 42_U3 & ... has a will of his/her own when listening and making music. & 3.70 & 0.98 \\
\hline 21_U3 & ... knows which music he/she likes and which he/she does not like. & 3.70 & 1.08 \\
\hline 1_U3 & ... has a musical ear, which means he/she can recognize simple melodies and tone progressions. & 3.70 & 0.90 \\
\hline
\end{tabular}

TABLE 2 | Fit indices of computed models.

\begin{tabular}{|c|c|c|c|c|c|}
\hline & BIC & TLI & CFI & RMSEA & SRMR \\
\hline 2-component PCA (following Hallam, varimax rotated) & 17520.93 & 0.778 & 0.790 & 0.074 & 0.070 \\
\hline 2-component PCA (parallel analysis, oblimin rotated) & 17518.47 & 0.778 & 0.790 & 0.074 & 0.070 \\
\hline 4-component PCA (according to grouping in 3-6-year-olds, oblimin rotated) & 17568.80 & 0.767 & 0.781 & 0.076 & 0.074 \\
\hline 4-component PCA (parallel analysis, under-3-year-olds, oblimin rotated) & 17481.65 & 0.793 & 0.805 & 0.072 & 0.070 \\
\hline
\end{tabular}

BIC, Bayesian Information Criterion; TLI, robust Tucker-Lewis Index; CFI, robust comparative fit index; RMSEA, robust root mean square error of approximation; SRMR, standardized root square residual.

The selected model is displayed in bold.

making music") or the ability to express oneself through music (e.g., "A child under 3 years who is musically skilled can invent melodies or rhythms, either with the voice, or by creating other sounds"). This component included factor loadings ranging from 0.40 to 0.57 and was termed Musical Communication.

The second component contained 15 items with loadings ranging from 0.31 to 0.85 . We named this component Enthusiasm and Motivation, because many high loading items described general interest in music (e.g., "A child under 3 years who is musically skilled has great enthusiasm for music") and the inherent motivation to become musically active (e.g., "A child under 3 years who is musically skilled often has the desire to make music"). Also associated with this component were items on creativity, playing with feeling, and having one's own taste in music.

Component 3 was interpreted as Adaptive Expressiveness. This component described the ability of a musical child to respond flexibly to different types of music, to appreciate different kinds of music and to react correspondingly in creative, emotional, and intellectual ways. It comprised factor loadings from 0.38 to 0.74 .

Component 4 was composed of 10 items (component loadings ranged from 0.34 to 0.75 ) focusing on musical abilities in a more specific sense (e.g., having a musical ear and a sense of timing, rhythm, and beat). It also contained items describing a set of more global abilities and behaviors (e.g., "A child under 3 years who is musically skilled has a wide range of different skills"; ". . . is able to combine hearing and sound production"). Therefore, we named the component Musical Abilities. Table 4 shows the mean ratings and the amount of explained variance by component.

\section{Constructing the MCQ_U3 Short Scale}

Grouping the items of the MCQ_U3 provided an informative classification suggesting four different facets of young children's musicality. In a next step, we constructed a short scale of the MCQ_U3 to obtain a more robust and practical measurement instrument for use in future studies.

The short scale was constructed using parallel analysis with PCA and subsequent oblimin rotation. Then, only items with component loadings of $>0.4$ were retained in order to reduce the number of items and maximize their discriminatory power. This resulted in a 2-component solution, which included 26 items and explained $51 \%$ of the variance (see Table 5 for the retained items and their respective loadings). The first component comprised a diverse set of items on perceptual and productive musical abilities and was therefore named Basic Musical Abilities (it contained items from the 3 components Musical Communication, Adaptive Expressiveness, and Musical Abilities of the 4-component solution). The second component included items on motivation, interest, and enthusiasm. Therefore, we kept the name Enthusiasm and Motivation.

\section{Professional Background and Musical Expertise Associated With Differences in the Evaluation of Musicality}

Through regression, we computed the component scores for all participants. Subsequently, we explored the influence of profession using a multivariate analysis of variance with professional background (professional educators, parents, and caregivers) as the independent variable and the two component scores as dependent variables. The test revealed no significant 
TABLE 3 | Items of the Musical Infant/Toddler Questionnaire (MCQ_U3), grouped by components.

\begin{tabular}{|c|c|c|c|c|c|c|c|}
\hline \multirow[b]{2}{*}{ Item no. } & \multirow{2}{*}{$\begin{array}{l}\text { MCQ_U3 item } \\
\text { A child under } 3 \text { years who is musically skilled... }\end{array}$} & \multirow[b]{2}{*}{$M$} & \multirow[b]{2}{*}{$S D$} & \multicolumn{3}{|c|}{ Component loading } & \multirow[b]{2}{*}{4} \\
\hline & & & & 1 & 2 & 3 & \\
\hline \multicolumn{8}{|c|}{ Component 1: Musical Communication } \\
\hline 2_U3 & ... can sing simple melody pieces. & 3.47 & 1.09 & 0.57 & 0.41 & -0.17 & 0.05 \\
\hline 30_U3 & ... can invent melodies or rhythms, either with the voice, or by creating other sounds. & 3.31 & 1.08 & 0.53 & 0.11 & 0.26 & 0.03 \\
\hline 3_U3 & ... is able to internalize simple sound sequences. & 3.44 & 0.96 & 0.47 & 0.11 & 0.08 & 0.36 \\
\hline 8_U3 & ... shows that he/she can capture patterns when dancing or making music. & 3.32 & 0.10 & 0.47 & 0.11 & 0.05 & 0.36 \\
\hline 27_U3 & $\begin{array}{l}\text {... can communicate with others through music by producing musical sounds, listening, } \\
\text { improvising, dancing, and understanding music. }\end{array}$ & 3.24 & 1.05 & 0.40 & 0.13 & 0.36 & 0.07 \\
\hline \multicolumn{8}{|c|}{ Component 2: Enthusiasm and Motivation } \\
\hline 41_U3 & ... has great enthusiasm for music. & 4.09 & 0.81 & 0.03 & 0.85 & 0.04 & -0.14 \\
\hline 38_U3 & $\ldots$ is interested in music. & 4.16 & 0.85 & -0.08 & 0.75 & 0.07 & 0.08 \\
\hline 43_U3 & ... enjoys the occupation with music. & 4.20 & 0.78 & 0.04 & 0.75 & 0.04 & -0.04 \\
\hline 14_U3 & ... enjoys music in his/her life, either by making music or by listening to it. & 4.05 & 0.87 & 0.07 & 0.72 & -0.24 & 0.22 \\
\hline 39_U3 & ... often has the desire to make music. & 3.67 & 0.93 & 0.01 & 0.67 & 0.10 & 0.02 \\
\hline 45_U3 & $\ldots$ has a great affinity for music. & 3.94 & 0.84 & -0.11 & 0.60 & -0.06 & 0.35 \\
\hline 15_U3 & ... enjoys music and appreciates sounds. & 3.88 & 0.89 & 0.05 & 0.56 & 0.18 & 0.03 \\
\hline 31_U3 & ... likes to spontaneously produce music or musical sounds. & 3.68 & 0.93 & 0.20 & 0.56 & 0.21 & 0.04 \\
\hline 29_U3 & $\begin{array}{l}\text {... has the desire to make music together with others so that they become a group - either by } \\
\text { singing along or moving to the music of others. }\end{array}$ & 3.76 & 0.99 & -0.07 & 0.51 & -0.21 & 0.35 \\
\hline 42_U3 & ... has a will of his/her own when listening and making music. & 3.70 & 0.98 & 0.23 & 0.51 & 0.15 & -0.03 \\
\hline 10_U3 & ... actively incorporates music into his/her world. & 3.49 & 0.98 & 0.24 & 0.50 & 0.27 & -0.02 \\
\hline 25_U3 & ... plays music with feeling. & 3.57 & 1.08 & 0.30 & 0.47 & 0.16 & 0.01 \\
\hline 32_U3 & $\ldots$ is creative when making music. & 3.57 & 1.03 & 0.38 & 0.42 & 0.18 & -0.07 \\
\hline 9_U3 & ... recognizes simple structural characteristics of music (e.g., loud-soft, slow-fast). & 3.64 & 0.93 & 0.40 & 0.41 & 0.03 & 0.08 \\
\hline 21_U3 & ... knows which music he/she likes and which he/she does not like. & 3.70 & 1.08 & 0.28 & 0.31 & 0.04 & 0.10 \\
\hline \multicolumn{8}{|c|}{ Component 3: Adaptive Expressiveness } \\
\hline 44_U3 & ... would rather express themselves through music than through other means. & 2.58 & 1.06 & 0.06 & 0.00 & 0.74 & -0.02 \\
\hline 17_U3 & ... appreciates different kinds of music. & 3.08 & 1.01 & -0.18 & 0.18 & 0.65 & 0.13 \\
\hline 26_U3 & ... perceives the basic mood or feelings conveyed by the music that he/she is listening to. & 3.10 & 0.99 & -0.02 & 0.08 & 0.64 & 0.21 \\
\hline 11_U3 & ... is able to react to the mood of a melody. & 3.22 & 1.03 & 0.06 & 0.03 & 0.64 & 0.23 \\
\hline 22_U3 & $\ldots$ is able to hear differences between different types of music. & 3.04 & 1.07 & 0.23 & -0.06 & 0.56 & 0.14 \\
\hline 46_U3 & A child is musical when music means something to him/her. & 3.23 & 1.12 & -0.37 & 0.32 & 0.51 & 0.08 \\
\hline 16_U3 & ... has an open mind with which he/she approaches and experiences all music. & 3.40 & 1.00 & -0.06 & 0.48 & 0.51 & -0.23 \\
\hline 18_U3 & ... recognizes different types of music (e.g., children's songs, pop, classical music). & 2.77 & 1.22 & 0.28 & -0.20 & 0.49 & 0.09 \\
\hline 13_U3 & ... is able to react creatively, emotionally and intellectually when listening to a piece of music. & 3.24 & 1.04 & 0.35 & 0.08 & 0.47 & 0.06 \\
\hline 19_U3 & ... reacts consciously to different aspects of music. & 3.26 & 1.03 & 0.39 & -0.12 & 0.46 & 0.15 \\
\hline 47_U3 & ... can immerse him-/herself in sounds. & 3.11 & 1.08 & 0.20 & 0.31 & 0.38 & 0.13 \\
\hline \multicolumn{8}{|c|}{ Component 4: Musical Abilities } \\
\hline 6_U3 & ... has a sense of timing and rhythm. & 3.41 & 1.04 & 0.14 & -0.04 & -0.01 & 0.75 \\
\hline 24_U3 & ... has good overall physical coordination. & 3.19 & 0.91 & -0.11 & 0.00 & 0.08 & 0.69 \\
\hline 36_U3 & ... has a wide range of different skills. & 3.35 & 0.96 & -0.08 & -0.10 & 0.28 & 0.58 \\
\hline 12_U3 & $\ldots$ is able to move according to the music. & 3.51 & 0.93 & -0.15 & 0.33 & 0.11 & 0.47 \\
\hline 7_U3 & ... has a feeling for the beat. & 3.26 & 1.01 & 0.22 & 0.00 & 0.22 & 0.46 \\
\hline 1_U3 & ... has a musical ear, which means he/she can recognize simple melodies and tone progressions. & 3.70 & 0.90 & 0.37 & 0.09 & 0.09 & 0.45 \\
\hline 35_U3 & ... is able to combine hearing and sound production. & 3.68 & 0.88 & 0.17 & 0.02 & 0.18 & 0.42 \\
\hline 4_U3 & ... has good hearing ability. & 3.63 & 0.90 & -0.04 & 0.23 & 0.21 & 0.42 \\
\hline 20_U3 & ... likes listening to music and finds matching gestures or movements. & 3.63 & 0.88 & 0.06 & 0.29 & 0.07 & 0.40 \\
\hline 5_U3 & ... has a good memory for patterns. & 3.28 & 0.98 & 0.17 & 0.29 & 0.18 & 0.34 \\
\hline
\end{tabular}

relationship between professional educators and parents and carers $\left[V=0.31, F(2,166)=2.63, p=0.08\right.$, partial $\left.\eta^{2}=0.031\right]$, suggesting that a professional background in child education did not influence the conceptions of musicality in infants/toddlers. However, the correlations between the Gold-MSI musical training subscale and the components Basic Musical Abilities 
TABLE 4 | Mean ratings grouped by components.

\begin{tabular}{|c|c|c|c|c|c|}
\hline & $M$ & $S D$ & $n$ & $\begin{array}{c}\text { Number of items } \\
\text { included }\end{array}$ & $\begin{array}{c}\text { Explained } \\
\text { variance (\%) }\end{array}$ \\
\hline $\begin{array}{l}\text { Musical } \\
\text { Communication }\end{array}$ & 3.36 & 0.77 & 174 & 5 & 9 \\
\hline $\begin{array}{l}\text { Enthusiasm } \\
\text { and Motivation }\end{array}$ & 3.81 & 0.64 & 174 & 15 & 18 \\
\hline $\begin{array}{l}\text { Adaptive } \\
\text { Expressiveness }\end{array}$ & 3.12 & 0.72 & 174 & 11 & 14 \\
\hline Musical Abilities & 3.47 & 0.62 & 174 & 10 & 11 \\
\hline
\end{tabular}

SD, Standard Deviation; $n$, number of participants.

( $r=0.26, p=0.001)$ and Enthusiasm and Motivation $(r=0.27$, $p<0.001$ ) of the MCQ_U3 short scale, were significant and of low to moderate strength, indicating that musical expertise is associated with slightly higher ratings on both components.

\section{Differences Between Conceptions of Musicality in Infants/Toddlers ( $<3$ Years) and Older Children (3-6 Years)}

Inspection of item overlap on the short scales (MCQ vs. MCQ_U3) showed that the component Musical Communication (MCQ) did not have any equivalent in the MCQ_U3. Furthermore, the components Analytical Understanding and Musical Abilities (MCQ) were combined into one component of the MCQ_U3 (Basic Musical Abilities). The component Enthusiasm and Motivation was highly similar in both short scales. The high content similarity was also reflected in a high Rand Index (a measure of similarity between two data clusterings; $\mathrm{RI}=0.83$, RI adjusted for chance $=0.65$ ). This means that items that were grouped into one component in the version for older children fall mostly into one component in the version for younger children as well.

\section{DISCUSSION}

This study explored the origins of musical behavior in early childhood. To this end, we investigated how German adults conceptualize musicality in children under the age of 3 years. More specifically, we were interested in which facets of the construct musicality are considered important by people with different professional and musical experience and whether these facets coincide with those that can be assessed using common musicality tests. Through PCA analysis, we identified four components of musicality in early childhood: Musical Communication, Enthusiasm and Motivation, Adaptive Expressiveness, and Musical Abilities. Interestingly, only this last component reflects traditional conceptions of musicality that strongly relate to aural abilities (we named this component Musical Abilities), whereas the three remaining components dealt with elements not traditionally captured by musical ability tests. Our results are largely consistent with previous findings on musicality in 3- to 6-year-old children (Buren et al., 2021). Thus, three components in the current study were broadly similar to those in the previous study of musicality in 3- to 6-year-old children (Musical Communication, Enthusiasm and Motivation, Musical Abilities). However, we were also able to show that the facets are not completely alike. Thus, the component Analytical Understanding of Music is not found in the under-3-year-olds. Instead, we found the facet Adaptive Expressiveness, which was not part of musicality in older children.

The Adaptive Expressiveness component was found to represent emotional facets of musicality. These include the ability to perceive and respond to the mood of musical stimuli. The component represents the ways in which children adapt their expressive reactions to different aspects of the music. In addition, items of this component indicate that children who are considered musical are open-minded to all types of music and recognize and appreciate different kinds of music. Analytical Understanding of Music, which was a component of musicality conceptions in older children (Buren et al., 2021), probably does not yet play a role in younger children because of developmental differences. Younger children seem to have a more spontaneous and intuitive access to music, whereas in older children the access to music may have become more analytical and includes a cognitive understanding as well.

On average, enthusiasm for music and the motivation to become musically active was rated by all participants as the most important sign of musicality in children under 3 years of age. This is in line with previous conceptions of child musicality in children older than 3 years (Buren et al., 2021). This suggests that enthusiasm for music is seen as a foundation for musical skill development. Although almost all infants generally show interest in musical stimuli and activities, interindividual differences in musical enthusiasm and motivation could be interpreted as indicators of musicality. These interindividual differences in musical interest might also be linked to the Big Five personality trait openness-to-experience which has been associated with aesthetic interest (McManus and Furnham, 2006) and musical engagement in both children and adults (Corrigall et al., 2013; Müllensiefen et al., 2014). The assumption that motivation predicts musical achievement is also evident in the observation that motivational factors are often used as criteria for selecting musical talent among students (Haroutounian, 2000). Motivation has also been conceptualized as a facet of musicianship in adulthood, recognizing the importance of deliberate effort in the development of musical expertise (Hallam, 2010).

Nevertheless, the measurement of motivational aspects has not yet been given much attention in the scientific assessment of musicality. Perhaps this is the result of methodological difficulties in the measurement of motivational aspects or is a reflection of the concern that motivation may be short-lived (Karma, 2007). However, because enthusiasm and motivation seem to be crucial components of musicality, we need valid measurements, questionnaires, or systematic observations to gain further insight into their importance for musical ability development.

The component Musical Abilities reflects traditional conceptions of musical ability relating to audiation, as assessed by traditional musicality tests. Examples include having a sense of timing and rhythm, a feeling for the beat, and a good musical ear. Additionally, some items describe the ability to move in time with music and find 
TABLE 5 | Short scale of the Musical Infant/Toddler Questionnaire grouped by components.

\begin{tabular}{|c|c|c|c|}
\hline \multirow[b]{2}{*}{ Item no. } & \multirow{2}{*}{$\begin{array}{l}\text { MCQ_U3 item } \\
\text { A child under } 3 \text { years who is musically skilled... }\end{array}$} & \multicolumn{2}{|c|}{ Component loading } \\
\hline & & 1 & 2 \\
\hline \multicolumn{4}{|c|}{ Component 1: Basic Musical Abilities } \\
\hline 19_U3 & ... reacts consciously to different aspects of music. & 0.78 & -0.19 \\
\hline 22_U3 & ... is able to hear differences between different types of music. & 0.75 & -0.11 \\
\hline 11_U3 & $\ldots$ is able to react to the mood of a melody. & 0.74 & -0.02 \\
\hline 3_U3 & ... is able to internalize simple sound sequences. & 0.72 & 0.03 \\
\hline 8_U3 & ... shows that he/she can capture patterns when dancing or making music. & 0.68 & 0.04 \\
\hline 7_U3 & ... has a feeling for the beat. & 0.67 & -0.05 \\
\hline 13_U3 & ... is able to react creatively, emotionally and intellectually when listening to a piece of music. & 0.67 & 0.04 \\
\hline 26_U3 & ... perceives the basic mood or feelings conveyed by the music that he/she is listening to. & 0.67 & 0.04 \\
\hline 1_U3 & ... has a musical ear, which means he/she can recognize simple melodies and tone progressions. & 0.66 & 0.06 \\
\hline 27_U3 & $\begin{array}{l}\text {... can communicate with others through music by producing musical sounds, listening, improvising, } \\
\text { dancing, and understanding music. }\end{array}$ & 0.65 & 0.09 \\
\hline 30_U3 & ... can invent melodies or rhythms, either with the voice, or by creating other sounds. & 0.65 & 0.04 \\
\hline $47 \_$U3 & ... can immerse him-/herself in sounds. & 0.58 & 0.25 \\
\hline 5_U3 & ... has a good memory for patterns. & 0.56 & 0.22 \\
\hline 9_U3 & ... recognizes simple structural characteristics of music (e.g., loud-soft, slow-fast). & 0.42 & 0.33 \\
\hline \multicolumn{4}{|c|}{ Component 2: Enthusiasm and Motivation } \\
\hline 41_U3 & $\ldots$ has great enthusiasm for music. & -0.11 & 0.90 \\
\hline 14_U3 & ... enjoys music in his/her life, either by making music or by listening to it. & -0.10 & 0.82 \\
\hline 38_U3 & $\ldots$ is interested in music. & 0.03 & 0.77 \\
\hline 43_U3 & ... enjoys the occupation with music. & 0.00 & 0.75 \\
\hline 39_U3 & $\ldots$ often has the desire to make music. & 0.04 & 0.69 \\
\hline 45_U3 & ... has a great affinity for music. & 0.05 & 0.68 \\
\hline 15_U3 & ... enjoys music and appreciates sounds. & 0.19 & 0.56 \\
\hline 31_U3 & ... likes to spontaneously produce music or musical sounds. & 0.34 & 0.55 \\
\hline 10_U3 & ... actively incorporates music into his/her world. & 0.37 & 0.48 \\
\hline 42_U3 & ... has a will of his/her own when listening and making music. & 0.28 & 0.47 \\
\hline 32_U3 & $\ldots$ is creative when making music. & 0.31 & 0.44 \\
\hline 25_U3 & ... plays music with feeling. & 0.37 & 0.42 \\
\hline
\end{tabular}

matching gestures and movements. This shows that musicality is not limited to perceptual musical skills, but is rather perceived as diverse and multiform, involving motor skills and the integration of skills across senses. Even in very young children our results demonstrate the complexity of musical behaviors.

The component Musical Communication focuses on using music for communicative purposes. To communicate musically, infants need a considerable "toolbox" for the perception and stimulation of communicative signals (Malloch, 2000). Hence, on the one hand, the component included items on the ability to capture and internalize musical stimuli; on the other hand it included the ability to invent melodies or rhythms, produce musical sounds, rhythmic movements, or sing.

Reducing the items for the short scale of the MCQ_U3 resulted in a 2-component model in which the Enthusiasm and Motivation component remained stable in content and the remaining three components were combined into one component (which we named Basic Musical Abilities). The component Basic Musical Abilities should thus be understood as something significantly more complex than what conventional measures of musical ability capture, which underpins the need for additional measures that cover a broader range of abilities and behaviors.

Using the short scale, we found that professional training in education or professional experience as an educator had no significant effect on the ratings of either component which stands in contrast to previous findings (although participants with more musical training gave slightly higher ratings on both components). In general, our results suggest that there is a high level of agreement about what constitutes musicality in young children.

Adult caregivers shape children's musical environment and their conception of childhood musicality forms the basis of their musical nurturing and education. Therefore, it is important to understand how adults view children's musicality, because musical interactions between caregiver and infant contribute to the infant's musical development in a meaningful way. Our study has shown that parents and carers are aware of motivational aspects being a key component in musical development. It remains unclear if they are aware of ways to foster their children's motivation. Many parents might be unaware of their own musicality and might rely on recorded music or institutional learning to develop their child's musical skills (Trehub, 2015). 


\section{Limitations and Future Directions}

Finally, potential limitations need to be considered. Our questionnaire was based on statements obtained from a qualitative study of conceptions of musicality in adults (Hallam and Prince, 2003). We conscientiously adjusted the statements to reflect conditions in childhood. However, because the original study focused on musicality in adulthood, the original responses may not have included all aspects of musicality in under-3-yearolds. Nevertheless, the high level of agreement with the selected statements showed that the used items, in general, reflected the conceptions of our sample.

The focus on social conceptions of musicality entails the limitation that the results may be restricted to a German population or Western music cultures. In particular, these conceptions may relate to children's experiences and opportunities. It has been shown that the musical abilities of infants/toddlers and preschoolers differ significantly and that parents and caregivers perceive this accordingly and adjust their musical endeavors (Politimou et al., 2018). Thus, the environment strongly influences the musical behaviors exhibited and, consequently, the conceptions of musicality. In addition, the selected age range ( $0-3$ years) is associated with large developmental changes. Some items were therefore not necessarily suitable for children across the full age range (some behaviors may not yet be exhibited by the youngest children). Here, a more precise differentiation could provide a clearer picture. Nevertheless, we think that our results are a first step toward capturing social constructs of musicality in very young children. Based on our results, however, finer classifications should be chosen for further studies and test development in order to better reflect developmental aspects.

Another future goal is to use the identified conceptions as a basis for examining childhood musicality and to explore whether this framework can be used to develop and validate test procedures as well as rating scales that reflect the social construct of musicality and, in so doing, trace the nature of musicality.

Hopefully, such test procedures will help to sketch out a more complete and comprehensive picture of musical development. Moreover, they could serve to identify individual differences in musical development between children.

At the moment we tend to draw our conclusions from sample averages rather than individual data (Sloboda, 2008). A key problem of these studies is, however, that their results tell us little about how individual developmental trajectories are shaped, because this information can get lost in aggregated values (Sloboda, 2008).

\section{Practical Implications}

The results of this study illustrate the complexity and multilayered nature of musicality - even among the very young. Furthermore, we were able to show that conceptions of musicality are differentiated and adapted to musical and general developmental levels. In line with previous studies, the results also point to the great importance of enthusiasm and motivation for musical development processes. Given that everyone has some degree of musical potential (Gordon, 1987; Gembris, 1997;
Honing, 2018), what can be done to help children to nurture their individual gifts? There is evidence that a musically enriched environment in which music is appreciated and enjoyed is critical for future development of musical ability (Shuter-Dyson, 1999) and may help children to develop a positive musical self-concept and motivation to develop their skills (Sloboda et al., 1994). The promotion of musical development must therefore not be limited to music lessons, but should explicitly include supposedly "ordinary" activities such as singing with the child (Papoušek, 1982; Trevarthen and Malloch, 2002). The importance of these experiences as learning opportunities may be greatly underestimated (Papoušek, 1982). Parents and caregivers (or educators) can thus promote musicality by interacting musically with children, stimulating their musical interests, and providing them with joyful musical experiences. Hence, early years musical development can be supported by acknowledging the spontaneous musical outputs of young children (e.g., rhythmic movements, cooperative singing) and responding to them. Such interactive forms of musical communication may be very effective in supporting children's enthusiasm and motivation for music (Flohr and Trevarthen, 2008).

\section{CONCLUSION}

This study is a first step toward enhancing a general understanding of conceptions of musicality in children under 3 years. We were able to show that already in early childhood, a multifaceted picture of musicality prevails. In particular, adaptive expressiveness, the ability to react flexibly to different musical stimuli, was shown for the first time to be a component of musicality. Furthermore, the results have highlighted the role of enthusiasm and motivation even in the youngest age group and have corroborated earlier findings that conceptions of musicality are broad and multifaceted. This reinforces the notion that traditional musical ability tests can only capture a limited portion of musicality and should therefore be supplemented by new assessment procedures, questionnaires, or observational measurement instruments. In the long run, a more comprehensive view and assessment of musicality may lead to an in-depth understanding of individual musical developmental trajectories and a better means of support for musical development.

\section{DATA AVAILABILITY STATEMENT}

The raw data supporting the conclusions of this article will be made available by the authors, without undue reservation.

\section{ETHICS STATEMENT}

The studies involving human participants were reviewed and approved by the Ethics Council of the Max Planck Society. The patients/participants provided their written informed consent to participate in this study. 


\section{AUTHOR CONTRIBUTIONS}

TR and VB contributed to the data collection. VB performed the literature search and drafted the manuscript. VB and DM

\section{REFERENCES}

Bentley, A. (1966). Musical Ability in Children and It's Measurement. Edinburg, TX: Harrap.

Berk, L. E. (2018). Development Though the Lifespan, 7th Edn. London: Pearson Education.

Blacking, J. (1971). "Towards a theory of musical competence," in Man: Anthropological Essays in Honour of O. F. Raum, ed. E. DeJager (Cape Town Struik), 19-34.

Blacking, J. (1995). Music, Culture and Experience. Chicago: University of Chicago Press.

Bukatko, D., and Daehler, M. W. (2004). Child Development: A Thematic Approach, 4th Edn. Boston, MA: Houghton Mifflin Harcourt.

Buren, V., Müllensiefen, D., Roeske, T., and Degé, F. (2021). What makes a child musical? conceptions of musical ability in childhood. Early Child Dev. Care 191, 1985-2000. doi: 10.1080/03004430.2020.1866566

Corrigall, K. A., Schellenberg, E. G., and Misura, N. M. (2013). Music training, cognition, and personality. Front. Psychol. 4:222. doi: 10.3389/fpsyg.2013.00222

Costa-Giomi, E., and Ilari, B. (2014). Infants' preferential attention to sung and spoken stimuli. J. Res. Music Educ. 62, 188-194. doi: 10.1177/ 0022429414530564

Davidson, L. (1994). "Songsinging by young and old: a developmental approach to music," in Musical Perceptions, eds R. Aiello and J. A. Sloboda (Oxford: Oxford University Press), 99-130.

de Vries, P. (2009). Music at home with the under fives: what is happening? Early Child Dev. Care 179, 395-405. doi: 10.1080/03004430802691914

Flohr, J. W., and Trevarthen, C. (2008). "Music learning in childhood: early developments of a musical brain and body," in Neurosciences In Music Pedagogy, eds W. Gruhn and F. F. Rauscher (Hauppauge, NY: Nova Biomedical Books), 53-99.

Forrester, M. A., and Borthwick-Hunter, E. (2015). Understanding the development of musicality: contributions from longitudinal studies. Psychomusicol.: Music Mind Brain 25, 93-102. doi: 10.1037/pmu0000086

Gembris, H. (1997). Historical phases in the definition of musicality. Psychomusicol.: J. Res. Music Cogn. 16, 17-25. doi: 10.1037/h009 4070

Gembris, H. (2017). in Grundlagen Musikalischer Begabung und Entwicklung. Issue 20, Vol. 5, ed. R.-D. Kraemer (Augsburg: Wißner).

Gooding, L., and Standley, J. M. (2011). Musical development and learning characteristics of students. Update: Appl. Res. Music Educ. 30, 32-45. doi: $10.1177 / 8755123311418481$

Gordon, E. E. (1965). Musical Aptitude Profile Manual. Boston, MA: Houghton Mifflin.

Gordon, E. E. (1979). Primary Measures of Music Audiation. Chicago, IL: GIA.

Gordon, E. E. (1982). Intermediate Measures of Music Audiation. Chicago, IL: GIA.

Gordon, E. E. (1987). The Nature, Description, Measurement and Evaluation of Music Aptitudes. Chicago, IL: G.I.A.

Gordon, E. E. (1989a). Advanced Measures of Music Audiation. Chicago, IL: GIA.

Gordon, E. E. (1989b). Audie: A Game for Understanding and Analysing Your Child's Musical Potential. Chicago, IL: GIA.

Gordon, E. E. (2007). Learning Sequences in Music: A Contemporary Music Learning Theory. Chicago, IL: GIA publications, Inc.

Hallam, S. (2010). 21St century conceptions of musical ability. Psychol. Music 38, 308-330. doi: 10.1177/0305735609351922

Hallam, S., and Papageorgi, I. (2016). Conceptions of musical understanding. Res. Stud. Music Educ. 38, 133-154. doi: 10.1177/1321103X1667 1037

Hallam, S., and Prince, V. (2003). Conceptions of musical ability. Res. Stud. Music Educ. 20, 2-22. doi: 10.1177/1321103X030200010101

Hallam, S., and Shaw, J. (2002). Constructions of musical ability. Bull. Council Res. Music Educ. 153, 102-106. performed the statistical analysis. FD and DM provided the critical revisions. All authors contributed equally in the project's conception and design of the questionnaire, contributed to the article, and approved the submitted version.

Hannon, E. E., and Trainor, L. J. (2007). Music acquisition: effects of enculturation and formal training on development. Trends Cogn. Sci. 11, 466-472. doi: 10.1016/j.tics.2007.08.008

Hannon, E. E., and Trehub, S. E. (2005). Tuning in to musical rhythms: infants learn more readily than adults. Proc. Natl. Acad. Sci. U.S.A. 102, 12639-12643. doi: $10.1073 /$ pnas.0504254102

Hargreaves, D. J., and Galton, M. (1992). "Aesthetic learning: psychological theory and educational practice," in 1992 N.S.S.E. Yearbook on the Arts in Education, eds B. Reimer and R. A. Smith (Chicago: N.S.S.E.), 124-150.

Haroutounian, J. (2000). Perspectives of musical talent: a study of identification criteria and procedures. High Abil. Stud. 11, 137-160. doi: 10.1080/ 13598130020001197

Honing, H. (2018). "Musicality as an upbeat to music: introduction and research agenda," in The Origins of Musicality, ed. H. Honing (Cambridge, MA: MIT Press), 3-20.

Horn, J. L. (1965). A rationale and test for the number of factors in factor analysis. Psychometrika 30, 179-185. doi: 10.1007/bf02289447

Ilari, B. (2005). On musical parenting of young children: musical beliefs and behaviors of mothers and infants. Early Child Dev. Care 175, 647-660. doi: $10.1080 / 0300443042000302573$

Ilari, B. (2018). Musical parenting and music education: integrating research and practice. Update: Appl. Res. Music Educ. 36, 45-52. doi: 10.1177/ 8755123317717053

Karma, K. (2007). Musical aptitude definition and measure validation: ecological validity can endanger the construct validity of musical aptitude tests. Psychomusicol.: J. Res. Music Cogn. 19, 79-90. doi: 10.1037/h0094033

Kirschner, S., and Tomasello, M. (2009). Joint drumming: social context facilitates synchronization in preschool children. J. Exp. Child Psychol. 102, 299-314. doi: 10.1016/j.jecp.2008.07.005

Malloch, S. N. (2000). Mothers and infants and communicative musicality. Music. Sci. 2, 29-57.

McManus, I. C., and Furnham, A. (2006). Aesthetic activities and aesthetic attitudes: influences of education, background and personality on interest and involvement in the arts. Br. J. Psychol. 97, 555-587. doi: 10.1348/ 000712606 X101088

McPherson, G. E. (2009). The role of parents in children-s musical development. Psychol. Music 37, 91-110. doi: 10.1177/0305735607086049

Mehr, S. A. (2014). Music in the home: new evidence for an intergenerational link. J. Res. Music Educ. 62, 78-88. doi: 10.1177/0022429413520008

Merriam, A. (1964). The Anthropology of Music. Evanston, IL: Northwestern University Press.

Müllensiefen, D., Gingras, B., Musil, J., and Stewart, L. (2014). The musicality of non-musicians: an index for assessing musical sophistication in the general population. PLoS One 9:e89642. doi: 10.1371/journal.pone.0089642

Murphy, C. (1999). How far do tests of musical ability shed light on the nature of musical intelligence? Br. J. Music Educ. 16, 39-50. doi: 10.1017/ s0265051799000133

Ockelford, A. (2013). Applied Musicology: Using Zygonic Theory to Inform Music Education, Therapy, and Psychology Research. Oxford: Oxford University Press.

Papoušek, M. (1982). The Mother Tongue Method of Music Education: Psychobiological Roots in Preverbal Parent-Infant Communication. ISME Yearbook, IX. Bristol: The Society, 103-108.

Politimou, N., Stewart, L., Müllensiefen, D., and Franco, F. (2018). Music@Home: a novel instrument to assess the home musical environment in the early years. PLoS One 13:e0193819. doi: 10.1371/journal.pone.0193819

Provasi, J., and Bobin-Bègue, A. (2003). Spontaneous motor tempo and rhythmical synchronisation in 21/2- and 4-year-old children. Int. J. Behav. Dev. 27, 220231. doi: 10.1080/01650250244000290

R Core Team (2019). R: A Language and Environment for Statistical Computing. $R$ Foundation for Statistical Computing. Vienna: Austria. Available online at: http://www.R-project.org/ (accessed May 25, 2020). 
Revelle, W. (2020). Psych: Procedures for Psychological, Psychometric, and Personality Research. R Package Version 2.0.7. Evanston, IL: Northwestern University.

Rosseel, Y. (2012). Lavaan: an R package for structural equation modeling. J. Stat. Softw. 48, 1-36. doi: 10.3389/fpsyg.2014.01521

Schaal, N. K., Bauer, A. K. R., and Müllensiefen, D. (2014). Der GoldMSI: replikation und Validierung eines Fragebogeninstrumentes zur Messung Musikalischer Erfahrenheit anhand einer deutschen Stichprobe. Musicae Sci. 18, 423-447. doi: 10.1177/1029864914541851

Schellenberg, E. G., Bigand, E., Poulin-Charronnat, B., Garnier, C., and Stevens, C. (2005). Children's implicit knowledge of harmony in Western music. Dev. Sci. 8, 551-566. doi: 10.1111/j.1467-7687.2005.00447.x

Seashore, C. E. (1919). The Psychology of Musical Talent. New York, NY: Silver, Burdett \& Company.

Seashore, C. E. (1967). Psychology of Music. Downers Grove, IL: Dover. (Originally published in 1938).

Serafine, M. L. (1988). Music as Cognition: The Development of Thought in Sound. New York, NY: Columbia University Press.

Shenfield, T., Trehub, S. E., and Nakata, T. (2003). Maternal singing modulates infant arousal. Psychol. Music 31, 365-375. doi: 10.1177/03057356030314002

Shuter-Dyson, R. (1999). "Musical ability," in The Psychology of Music, 2nd Edn. ed. D. Deutsch (San Diego, CA: Academic Press), 627-651.

Siegler, R., DeLoache, J., Eisenberg, N., Saffran, J., and Leaper, C. (2014). How Children Develop. New York, NY: Worth Publishers.

Sloboda, J. (2008). The ear of the beholder. Nature 454, 32-33. doi: 10.1038/ $454032 \mathrm{a}$

Sloboda, J., Davidson, J. W., and Howe, M. J. A. (1994). Is everyone musical? Psychologist 7, 349-354.

Stadler Elmer, S. (2011). Human Singing: towards a developmental theory. Psychomusicol.: Music Mind Brain 21, 13-30. doi: 10.1037/h0094001

Stadler Elmer, S. (2012). Characteristics of early productive musicality. Problems Music Pedagogy 10, 9-23. doi: 10.5167/uzh-95057

Swanwick, K., and Tillman, J. (1986). The sequence of musical development: a study of children's composition. Br. J. Music Educ. 3, 305-339. doi: 10.1017/ S0265051700000814

Trainor, L. J., and Unrau, A. (2011). "Development of pitch and music perception," in Springer Handbook of Auditory Research: Human Auditory Development, eds L. Werner, R. R. Fay, and A. N. Popper (Berlin: Springer), 223-254. doi: 10.1007/978-1-4614-1421-6_8

Trehub, S., and Hannon, E. (2006). Infant music perception: domain-general or domain-specific mechanisms? Cognition 100, 73-99.

Trehub, S. E. (2003). The developmental origins of musicality. Nat. Neurosci. 6, 669-673. doi: 10.1038/nn1084

Trehub, S. E. (2015). "Infant musicality," in The Oxford Handbook of Music Psychology, eds S. Hallam, I. Cross, and M. Thaut (Oxford: Oxford University Press).
Trehub, S. E., Becker, J., and Morley, I. (2015a). Cross-cultural perspectives on music and musicality. Philosop. Trans. R. Soc. B: Biol. Sci. 370:20140096. doi: 10.1098/rstb.2014.0096

Trehub, S. E., Ghazban, N., and Corbeil, M. (2015b). Musical affect regulation in infancy. Ann. N. Y. Acad. Sci. 1337, 186-192. doi: 10.1111/nyas.12622

Trehub, S. E., Hill, D. S., and Kamenetsky, S. B. (1997a). Parents' sung performances for infants. Can. J. Exp. Psychol. 51, 385-396. doi: 10.1037/1196-1961.51.4.385

Trehub, S. E., Unyk, A. M., Kamenetsky, S. B., Hill, D. S., Trainor, L. J., Henderson, J. L., et al. (1997b). Mothers' and fathers' singing to infants. Dev. Psychol. 33, 500-507. doi: 10.1037/0012-1649.33.3.500

Trevarthen, C., and Malloch, S. N. (2002). Musicality and Music Before Three: Human Vitality and Invention Shared with Pride. Zero to Three. Edinburg: The University of Edinburg, 0-18.

Valerio, W. H., Reynolds, A. M., Morgan, G. B., and McNair, A. A. (2012). Construct validity of the children's music-related behavior questionnaire. J. Res. Music Educ. 60, 186-200. doi: 10.1177/0022429412444450

Weiss, M. W., Bissonnette, A. M., and Peretz, I. (2021). The singing voice is special: persistence of superior memory for vocal melodies despite vocal-motor distractions. Cognition 213:104514. doi: 10.1016/j.cognition.2020.104514

Weiss, M. W., Schellenberg, G. E., Trehub, S. E., and Dawber, E. J. (2015). Enhanced processing of vocal melodies in childhood. Dev. Psychol. 51, 370-377. doi: 10.1037/a0038784

Weiss, M. W., Trehub, S. E., and Schellenberg, E. G. (2012). Something in the way she sings: enhanced memory for vocal melodies. Psychol. Sci. 23, 1074-1078. doi: $10.1177 / 0956797612442552$

Wing, H. D. (1981). Standardised Tests of Musical Intelligence. Slough: NFERNELSON.

Young, S. (2008). Lullaby light shows: everyday musical experience among undertwo-year-olds. Int. J. Music Educ. 26, 33-46. doi: 10.1177/0255761407085648

Conflict of Interest: The authors declare that the research was conducted in the absence of any commercial or financial relationships that could be construed as a potential conflict of interest.

Publisher's Note: All claims expressed in this article are solely those of the authors and do not necessarily represent those of their affiliated organizations, or those of the publisher, the editors and the reviewers. Any product that may be evaluated in this article, or claim that may be made by its manufacturer, is not guaranteed or endorsed by the publisher.

Copyright (c) 2021 Buren, Müllensiefen, Roeske and Degé. This is an open-access article distributed under the terms of the Creative Commons Attribution License (CC BY). The use, distribution or reproduction in other forums is permitted, provided the original author(s) and the copyright owner(s) are credited and that the original publication in this journal is cited, in accordance with accepted academic practice. No use, distribution or reproduction is permitted which does not comply with these terms. 